\title{
THE REPLACEMENT OF STREPTOMYCIN-RESISTANT COLIFORM BACTERIA IN THE STOOLS BY STREPTOMYCIN-SENSITIVE VARIANTS DURING AND FOLLOWING THE CESSATION OF STREPTOMYCIN THERAPY 1,2
}

\author{
BY MORTON HAMBLRGER AND JEROME R. BERMAN \\ (From The Department of Medicine, College of Medicinc, Uniz'crsity of Cincinnati, \\ (incinnati)
}

(Submitted for publication December 9, 1949; accepted, January 23, 1950)

Though the appearance of streptomycin-resistant bacteria during the course of streptomycin therapy has been abundantly demonstrated (1), less attention has been paid to the ultimate fate of these resistant forms. Our attention was drawn to this problem during an investigation of the development of streptomycin-resistant variants among the normally commensal but potentially pathogenic flora of the body. Though streptomycin-resistant coliform bacilli are almost uniformly absent from the stools of human beings who have not received streptomycin, they frequently appear during the intramuscular administration of streptomycin for certain types of tuberculosis (2) and during its oral administration for various purposes.

Our investigations have shown that in many cases the normally "sensitive" coliforms were replaced during treatment by resistant strains of the same species. However, when streptomycin was discontinued, and in special instances, even before the drug was discontinued, sensitive coliforms began to reappear and finally crowcled out the resistant variants altogether. It is the purpose of this paper to present the data dealing with this replacement of resistant by sensitive strains.

\section{METHODS}

\section{A. Selection of cases}

The 11 patients studied were hospitalized on the medical wards of the Cincinnati General Hospital. They included two cases of miliary tuberculosis, seven cases of tuberculous peritonitis, and two cases of typhoid fever.

\footnotetext{
1 This investigation was supported by the Division of Research Grants and Fellowships, National Institutes of Health, Bethesda, Md., by Merck and Company, and by the Holmes Research Fund.

$\because$ This paper was presented in part at the Second $\mathrm{Na}$ tional Antibiotics Conference in Washington, D. C., April 11, 1949; an abstract was published in the Journal of Clinical Investigation, 1949, Vol. 28, page 1042.
}

\section{B. Stool cultures on "plain" and "streptomycin" plates}

Approximately 0.5 gram stool was emulsified in $5 \mathrm{cc}$. $0.9 \%$ saline solution. One standard $3 \mathrm{~mm}$. loopful of this suspension was then streaked on each of two eosinmethylene blue agar plates, one of which was prepared so as to contain $100 \mu \mathrm{g}$. streptomycin per cc. of medium (henceforth referred to as the streptomycin plate), and the other no streptomycin (henceforth referred to as the plain plate). The plates were incubated at $37^{\circ} \mathrm{C}$., and examined at the end of 20 to 24 hours. The approximate number of colonies of coliform bacteria was recorded as + to ++++ . Plates showing no growth were reincubated and read at 48 and 72 hours. Many of the plates were photographed.

One to ten colonies were picked from most plates for determination of their streptomycin resistance by a serial dilution test. In some cases, 12 colonies selected at random from the plain plate were restreaked on a plate containing $100 \mu \mathrm{g}$. streptomycin per cc., incubated overnight at $37^{\circ}$ and examined for growth the next morning. This technique was employed as a rapid means of determining the approximate number of "sensitive" and "resistant" colonies on a plain plate.

\section{Serial dilution tests for streptomycin resistance}

One-half cc. of a solution of commercial streptomycin hydrochloride in sterile distilled water was placed in each of two $13 \times 100 \mathrm{~mm}$. tubes. One-half cc. sterile tryptone broth was added to the second tube, and twofold serial dilutions in broth made until the 11th tube was reached. One-half $\mathrm{cc}$. sterile broth containing no streptomycin was placed in the 12 th tube. One and onehalf cc. of a 1:100 dilution of an 18 hour culture of the unknown organism was added to each tube. The tests were incubated at $37^{\circ} \mathrm{C}$. for 24 hours. The lowest concentration of streptomycin in which no macroscopic growth occurred was recorded as the endpoint.

Streptomycin solutions of two strengths were employed. The "strong" solution, used in testing strains suspected of being resistant, contained 2,500 $\mu \mathrm{g}$. per cc. of medium as the final concentration in the first tube. The "weak" solution contained $5 \mu \mathrm{g}$. per cc. under the same conditions. The solutions were prepared under 


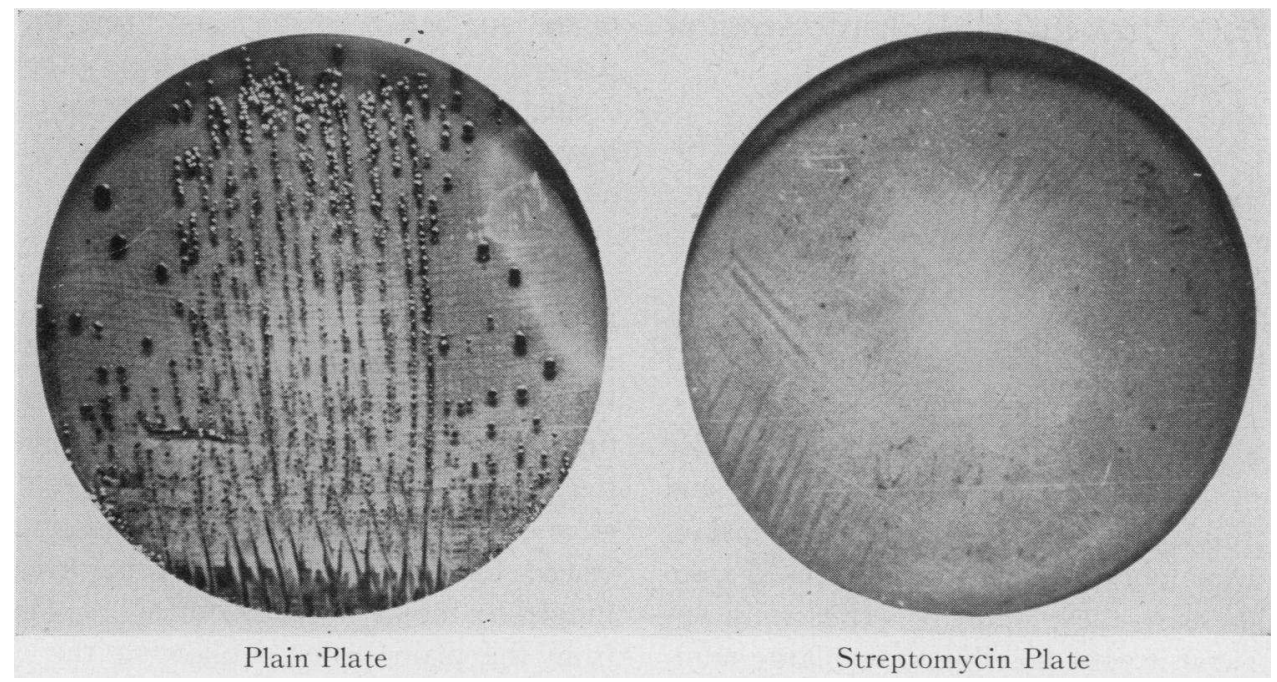

Fig. 1. Culture of Stool Suspension from an Untreated Patient on Plain and Streptomycin Plates

sterile conditions from the sterile vials supplied by the manufacturer, ${ }^{3}$ and were not autoclaved or filtered.

As a control upon the streptomycin, a culture of known sensitivity, B. Circulans, was tested along with the unknown strains.

Definition of "sensitive" and "resistant." Sensitive strains were those whose growth was inhibited by $5 \mu \mathrm{g}$. streptomycin or less. Highly resistant strains were those requiring from 156 to more than $2,500 \mu \mathrm{g}$. for growth inhibition. Strains whose growth was inhibited by more than 5 and less than $156 \mu \mathrm{g}$. were considered moderately resistant.

More than $95 \%$ of the strains encountered in this study fell into the categories of sensitive or highly resistant.

Identification of coliform bacteria. Colonies 1 to 2 $\mathrm{mm}$. in diameter showing a metallic sheen were classified as E. coli. Larger mucoid colonies exhibiting slimy growth were classified as Aerobacter aerogenes. Colonies 1 to $4 \mathrm{~mm}$. in size, relatively flat and dry but exhibiting no sheen and definitely not mucoid, were classified as paracolon bacilli.

Representative colonies from each patient were further identified by the "IMVIC" reactions as described by Parr (3). This series of biochemical tests is described in standard textbooks of bacteriology, and includes the indol, methyl red, Voges-Proskauer, and citrate reactions. $E$. coli gives positive reactions in the first two tests, but negative reactions in the second two. $A$. acrogenes reacts exactly oppositely.

\section{RESULTS}

Absence of resistant coliforms in stool cultures of patients zeho had not received streptomycin

Cultures were made of 57 specimens from 52 patients who had not received streptomycin at any

\footnotetext{
${ }^{3}$ Kindly donated by Merck and Company.
}

time. Cases of tuberculosis, rheumatic fever, brucellosis, cancer, urinary tract infection, pneumonia, and salmonellosis were included. Figure 1, which is a photograph of a typical pair of plates of a stool culture from an untreated patient, shows the luxuriant growth of coliforms on the plain plate, and absence of growth on the streptomycin plate. Determination of the streptomycin sensitivity by serial dilution tests of 81 colonies picked from plain plates of untreated patients revealed inhibition of growth by 0.6 to $2.5 \mu \mathrm{g}$. streptomycin per cc. No resistant colonies were ever encountered. All but one of the 57 stool suspensions which grew so luxuriantly on the plain plates failed to grow, even after 72 hours' incubation, on the streptomycin plates.

The only case where streptomycin-resistant coliforms were found in the stool prior to the administration of streptomycin was that of a patient with tuberculous peritonitis and Addison's Disease. In this case, growth on the plain plate was recorded as ++++ (i.e., hundreds of colonies), and ten colonies picked at random required only $0.6-1.25 \mu \mathrm{g}$. for growth inhibition. However, three colonies appeared on the streptomycin plate, all of which grew luxuriantly in $1,250 \mu \mathrm{g}$. when assayed in the serial dilution test. It was of interest that within less than 24 hours after the first dose of streptomycin given this patient, highly resistant coliforms appeared in very large numbers 
and completely replaced the original sensitive strains.

\section{Appearance of resistant variants during strepto- mycin therapy}

Streptomycin-resistant coliforms replaced the normally sensitive strains in the stools of 11 patients receiving streptomycin, nine by the intramuscular route and two both intramuscularly and by mouth. This was demonstrated in two ways. First, there was equal growth, as judged by size and number of colonies, on both the plain and streptomycin plates. Second, only highly resistant coliforms were found among colonies picked from the plain plates of these patients. It is fully appreciated that if it were possible to test large numbers of individual colonies from the plain plates, an occasional sensitive colony might have been found. For practical purposes, however, it seems correct to state that during treatment, in these patients, streptomycin-sensitive coliforms had disappeared from the stools.

Reappearance of streptomycin-sensitive coliforms after discontinuance of streptomycin

Similarly, the reappearance of streptomycinsensitive variants after their replacement by resistant forms was demonstrated in two ways: 1 ) the finding of sensitive strains among colonies picked from the plain plate, and/or 2) diminution of the number of colonies appearing on the streptomycin plate. Often these two processes proceeded simultaneously, but sometimes sensitive forms began to appear on the plain plate before any decrease of the number of colonies was noted on the streptomycin plate.

Under certain conditions (2) streptomycinresistant coliforms appear in the stools of patients who receive the antibiotic by the intramuscular route only. In nine patients where the opportunity presented itself to study the balance between sensitive and resistant variants during and after therapy, highly resistant variants had replaced the sensitive forms during treatment, as judged by the in vitro resistance of colonies picked from the plain plates. Following the discontinuance of streptomycin, sensitive flora began to return in every case. The ascendancy of the sensitive coliforms continued until finally, in all but two cases, no resistant variants could be detected. In one of these two, a single resistant colony was found a year after the end of treatment. In the other, streptomycin-resistant paracolon bacilli persisted in large numbers for at least four months after the patient had received no streptomycin.

The balance between sensitive and resistant strains in two such cases is illustrated in Figures 2 and 3. Figure 2 was prepared from the data of a patient with miliary tuberculosis. During treatment, growth on plain and streptomycin plates

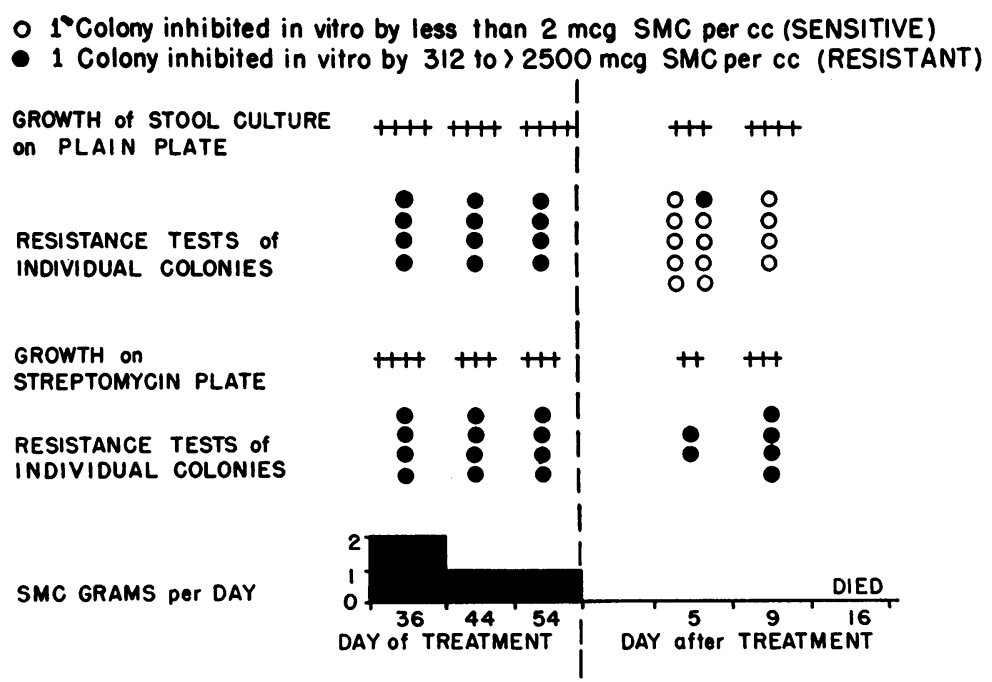

Fig. 2. Reappearance of Streptomycin-Sensitive Coliform Bacteria in Stool of a Patient with Miliary Tuberculosis Foldowing Refusal. of Further Treatment 
O 1 Colony inhibited in vitro by less than 2 mag SMC per cc (SENSITIVE)

- 1 Colony inhibited in vitro by 312 to 2500 mcg SMC per cc (RESISTANT)

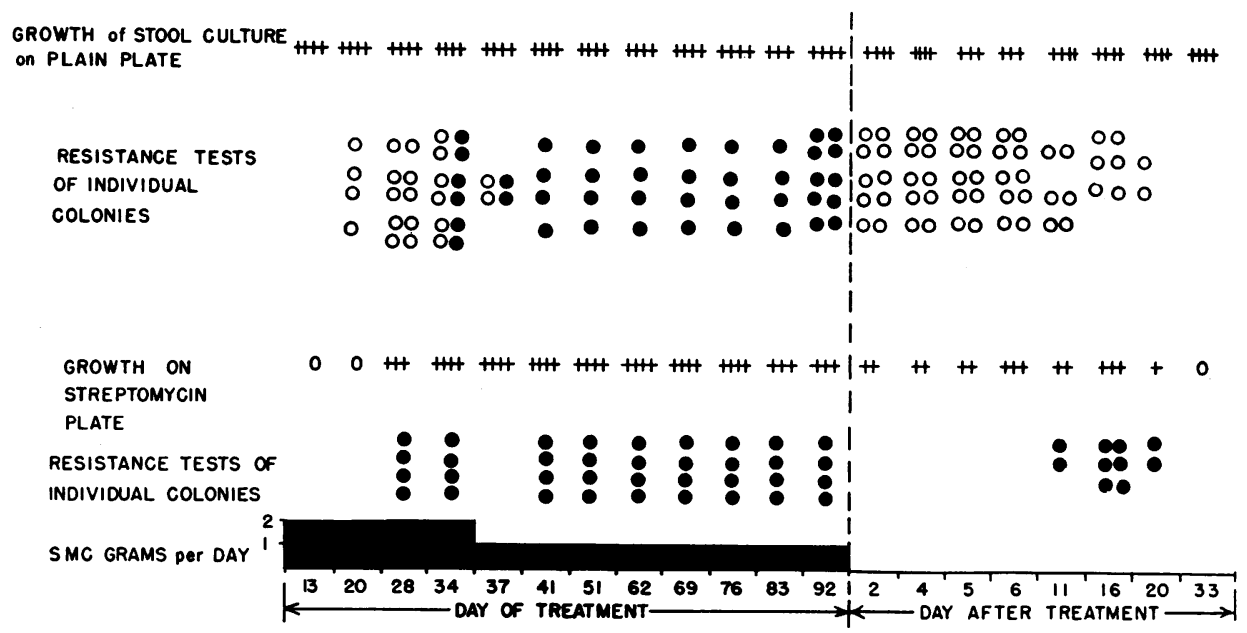

Fig. 3. Reappearance of Streptomycin-Sensitive Coliform Bacteria in Stool of a Patient with Tuberculous Peritonitis and Gradual. Disappearance of Resistant Variants Following Discontinuance of Treatment

was approximately equal, and all colonies picked from either plate were highly resistant to streptomycin. After 54 days of treatment, during which time the patient became gradually worse, he refused to take any more intramuscular injections. Five days later a stool specimen showed that though many resistant coliforms were still present on the streptomycin plate, nine of ten colonies picked from the plain plate were highly sensitive. In this case, the reappearance of sensitive strains was detected only by the study of individual colonies picked from the plain plate.

Figure 3 represents the events in a case of tuberculous peritonitis. During the first three

TABLE I

Replacement of streptomycin-resistant coliforms in the stool by sensitive variants after cessation of orally administered streptomycin

Stool cultures from a case of typhoid fever

\begin{tabular}{|c|c|c|c|c|c|}
\hline \multirow{2}{*}{$\begin{array}{l}\text { Date } \\
1948\end{array}$} & \multicolumn{2}{|c|}{ Plain plate } & \multicolumn{2}{|c|}{ Streptomycin plate } & \multirow{2}{*}{ Treatment } \\
\hline & E. coli & A. aerogenes & E. coli & A. aerogenes & \\
\hline $26 \mathrm{May}$ & $+++t$ & 0 & 0 & 0 & \\
\hline 4 June & ++++ & 0 & 0 & 0 & \\
\hline 17 June & $\begin{array}{c}0.6,1.2,0.6,0.6 \\
+++\end{array}$ & ++ & +++ & ++ & $\begin{array}{l}\text { June } 9-23 \text {, inclusive: } \\
\text { Streptomycin } 0.5 \text { gram }\end{array}$ \\
\hline $\begin{array}{l}19 \text { June } \\
21 \text { June }\end{array}$ & $\begin{array}{c}1250,1250 \\
++ \\
+\end{array}$ & $\begin{array}{c}2500,2500 \\
+ \\
++ \\
1250,1250 \\
0.6,1250\end{array}$ & $\begin{array}{c}625,625 \\
++ \\
+\end{array}$ & $\begin{array}{c}1250,1250 \\
+ \\
++ \\
2500,1250 \\
25002500\end{array}$ & $\begin{array}{l}\text { every } 8 \text { hours, intra- } \\
\text { muscularly. } \\
\text { June 16-23, inclusive: } \\
\text { Streptomycin } 0.5 \text { gram }\end{array}$ \\
\hline 25 June & $\begin{array}{c}++ \\
0.6,1250\end{array}$ & $\begin{array}{c}++ \\
1250,1250\end{array}$ & ++ & & \\
\hline 30 June & $\frac{+++}{1.2,0.6,0.3,0.6}$ & + & ++ & $\begin{array}{c}2500,2500 \\
++ \\
1250,1250\end{array}$ & \\
\hline $\begin{array}{l}17 \text { July } \\
27 \text { July } \\
21 \text { Aug. }\end{array}$ & $\begin{array}{l}++++ \\
++++ \\
++++\end{array}$ & $\begin{array}{l}0 \\
+ \\
+\end{array}$ & $\begin{array}{l}0 \\
0 \\
0\end{array}$ & $\begin{array}{c}1250,1250 \\
0 \\
0 \\
0\end{array}$ & \\
\hline
\end{tabular}

The amount of streptomycin, in $\mu$ g. per cc., required to inhibit the growth of individual strains picked from the plates is indicated by the numbers under the reading for each plate. 
weeks of treatment, no streptomycin-resistant coliforms were encountered. A specimen of stool obtained on the 28th day showed heavy growth on the streptomycin plate, though ten colonies picked from the plain plate were still sensitive. The next two specimens revealed the presence of resistant and sensitive variants on the plain plate in about equal numbers, but the following seven revealed only resistant forms. By the second day after cessation of treatment sensitive coliforms had replaced the resistant strains on the plain plate, though the persistence of resistant forms was easily demonstrated by streptomycin plates for nearly three weeks. After that time, resistant variants could no longer be detected.

The stool cultures in a case of typhoid fever are summarized in Table I. During combined oral and intramuscular treatment, highly resistant $E$. coli and $A$. aerogenes largely replaced the original sensitive strains, though in this instance $25 \%$ of the colonies picked from the plain plate during treatment remained sensitive to strepto- mycin. This finding proves that though during treatment the resistant variants may predominate, they do not always completely crowd out the sensitive forms. One week after streptomycin had been discontinued, resistant variants were not found on the plain plate, and 17 days later they had disappeared from the streptomycin plate.

Similar findings were encountered in another case of typhoid fever.

\section{Reappearance of streptomycin-sensitive coliforms during improvement under streptomycin therapy}

This interesting phenomenon was observed in three cases of tuberculous peritonitis, where streptomycin-resistant coliforms had replaced the original sensitive strains, during the intramuscular administration of streptomycin.

The events in the first case are illustrated graphically in Figures 4 and 5 . The patient was a young colored man with tuberculous peritonitis, pleuritis, and minimal pulmonary tuberculosis, whose streptomycin therapy was started before a
3 June '48

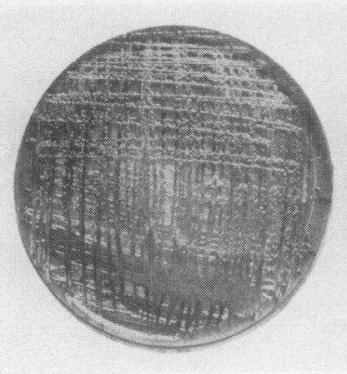

Plain

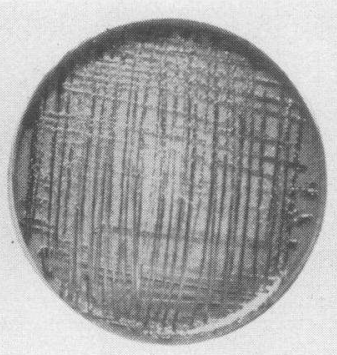

Streptomycin

29 June' 48

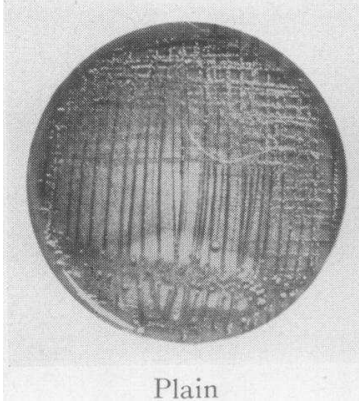

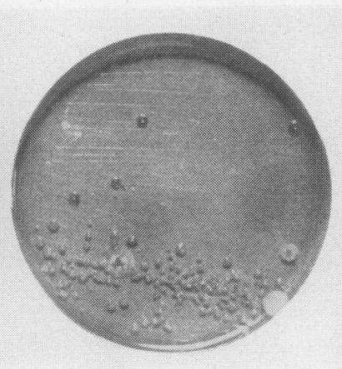

Streptomycin

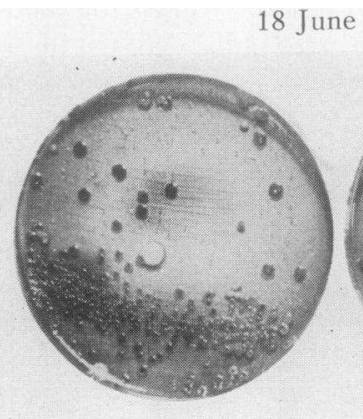

Plain

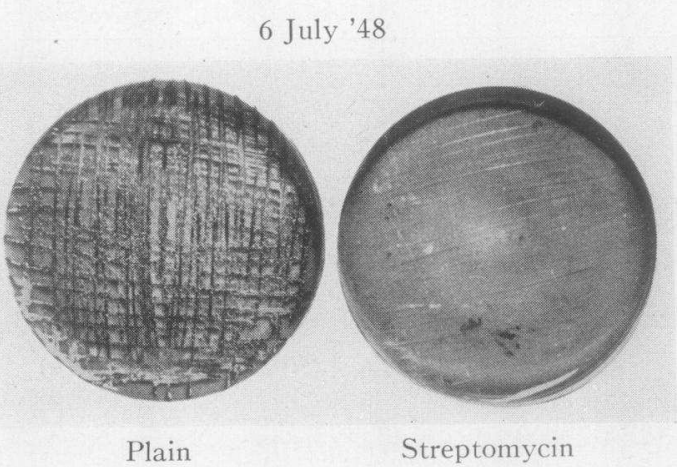

Figi. 4. Photograpis of Pairs of Stool Culture Plates Illustrating Replacement of Resistant by Sensitive Coliforms During Streptomycin Treatment

The June 3rd plates were made during the fourth week of treatment; the June 18th plates during the sixth week; the June 29th plates during the seventh week; and the July 6th plates during the ninth week.

Some of the photographs were made after the plates had been kept in the refrigerator as long as three weeks. 
O 1Colony inhibited in vitro by less than 2 mag SMCper cc (SENSITIVE)

- 1 Colony inhibited in vitro by 312 to $) 2500$ mcg SMC per CC (RESISTANT)

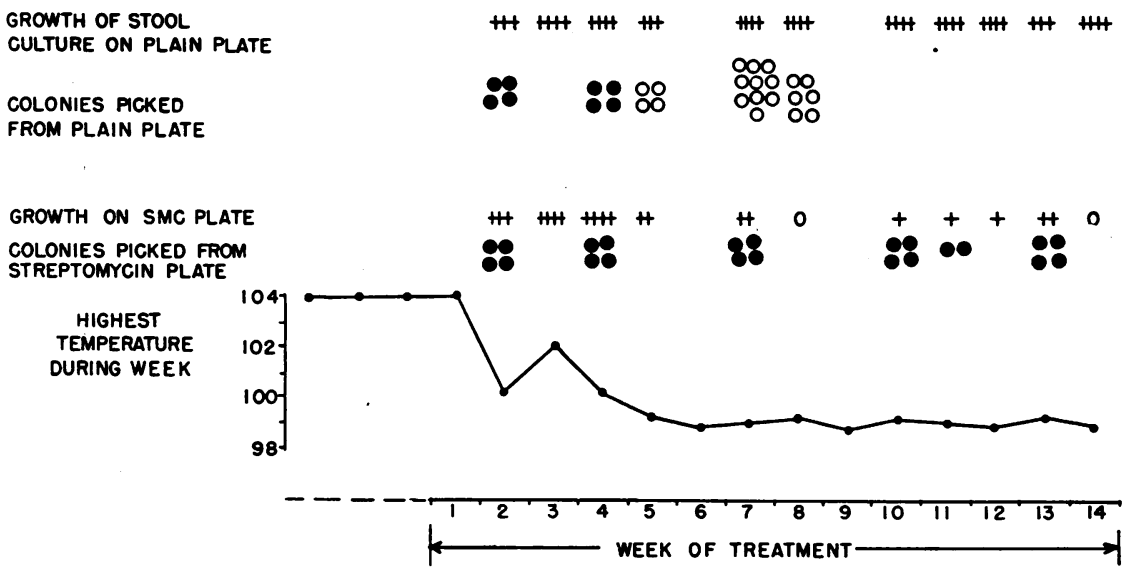

Fig. 5. Reappearance of Streptomycin-Sensitive Coliform Bacilli and Gradual Disappearance of Resistant Variants During Treatment of a Case of Tuberculous Peritonitis

Data from the same case as Figure 4.

stool culture was obtained. A specimen studied on the tenth day of treatment revealed heavy growth of $E$. coli and $A$. aerogenes on both plain and streptomycin plates, with only resistant colonies found on either plate. During the fifth week of therapy, when the patient had shown great clinical improvement, it was noted that the macroscopic growth on the streptomycin plate was somewhat less than on the plain plate. Much to our surprise, four colonies picked from the latter were highly sensitive to streptomycin. From that time on, growth on the streptomycin plate continued to decrease in amount, and only sensitive colonies were recovered from plain plates.

In the second case, resistant forms appeared after one week of treatment, persisted approximately two weeks, but were never again encountered in any of five specimens obtained over a six week period.

In the third case, highly resistant coliforms replaced the original sensitive forms in a single specimen during the seventh week of treatment. Four subsequent cultures revealed only sensitive strains.

Interval between last dose of streptomycin and reappearance of sensitive coliforms

In eight cases, the resistant variants continued to supplant the sensitive forms until streptomycin was discontinued. In these cases, either the first or the second specimen obtained during the posttreatment period revealed the presence of sensitive coliforms, the intervals between the last dose and the first specimen revealing sensitive strains being $3,3,5,7,7,25,110$, and 358 days.

For variable periods, both sensitive and resistant forms were recovered from the same stool specimen. Sometimes the resistant variants were detected only by the streptomycin plate, but other times they were found among colonies picked from the plain plate. In all but two cases, the resistant variants had disappeared entirely by the end of two months. 'In one case, a single resistant colony grew on a plate made a year after no streptomycin had been administered.

Passage of resistant colonies through streptomycinfree broth and untreated mice

Eight strains, resistant to $1,250-2,500 \mu \mathrm{g}$. streptomycin, from four patients, were passed 25 to 100 times through tryptone broth free of streptomycin. The streptomycin resistance was measured after each 25 passages. In no case was there any loss of resistance following passage in streptomycin-free medium.

One of the strains was passed serially by intraperitoneal inoculation through 15 untreated white mice and tested after each passage. Blood culture 
strains were used for testing. This strain remained as resistant after 15 passages as it had been originally.

These experiments inclicate that the replacement of resistant by sensitive strains in the patients studied was not due either to loss of resistance ("back mutation") or to the need for streptomycin as a metabolic requirement.

\section{DISCUSSION}

It has been well established that during the administration of antibiotics, the balance of the normal flora of the body is profoundly altered. In the throat, for example, an overgrowth of gram negative species usually persists as long as penicillin is administered, though after the drug is stopped, the previous ecological balance is restored and gram positive species return $(4,5)$. The observations reported in this paper, however, bear on the balance between streptomycin-sensitive and streptomycin-resistant variants within the same species, though in the absence of specific serological studies, one cannot assert that the sensitive and resistant variants we have studied may not be different strains within the species.

The reasons for the replacement of resistant by sensitive forms are not clear. The evidence indicates that the phenomenon is one of replacement rather than reversion to sensitive or development of "streptomycin-dependence." That the sensitive variants are not entirely eradicated during treatment seems highly likely, even in the cases where they were not demonstrated by the techniques employed.

The usual tests for the demonstration of streptomycin-resistant bacteria do not detect the presence of sensitive variants among resistant forms (6). In some studies, however $(7,8)$, the concomitant presence of resistant and sensitive tubercle bacilli in the same culture has been demonstrated. Thus, Pyle (8) showed that during the period of appearance of streptomycin-resistant tubercle bacilli in the sputum of treated patients, both sensitive and resistant variants may be present at the same time. She did not study sputum after the discontinuance of streptomycin. D'Esopo (7) has also found tubercle bacilli of varying degrees of resistance in single sputum samples of patients with pulmonary tuberculosis.
Most of the available evidence indicates that when resistant variants of a pathogenic microorganism replace the sensitive parent strain in the tissue it has invaded, the resistant variants usually remain for an indefinite period. Thus, Steenken and Wolinsky (9) found that when sputum yielded tubercle bacilli which had become resistant to $1,000 \mu \mathrm{g}$. streptomycin, sputa from the same patients 10 to 20 months after the cessation of treatment also yielded bacilli resistant to $1,000 \mu \mathrm{g}$. This suggests that the factors which determine the ecological balance between sensitive and resistant variants of a pathogen in the tissues may not be the same as those determining the balance between the variants within a commensal species.

\section{SUMMARY}

1. The relative numbers of streptomycin-sensitive and streptomycin-resistant coliform bacilli in the stools of patients receiving streptomycin intramuscularly or by mouth were studied in 11 patients during and after treatment. In eight of these cases, though resistant variants crowded out the original sensitive forms during treatment, the discontinuance of streptomycin was rapidly followed by the reappearance of streptomycinsensitive coliforms. Over a period of weeks or months, the resistant variants tended to disappear entirely.

2. In one case where resistant variants had crowled out the sensitive coliforms, the latter replaced the resistant strains during streptomycin therapy, coincident with clinical improvement. In two similar cases resistant forms appeared transiently, but disappeared while streptomycin was still being administered.

3. The return of the pre-treatment ecological balance within the same species could not be explained by reversion of the resistant forms to sensitive nor by the need for streptomycin as a metabolic requirement by the resistant coliforms, since "fast" variants maintained their high degree of resistance after 100 passages through streptomycin-free broth and 15 untreated mice.

\section{ACKNOWLEDGMENT}

Valuable technical assistance was rendered by Miss Angelina Fabrizio, Mrs. Jeanne Fife, and Miss Dorothy Muething. 


\section{BIBLIOGRAPHY}

1. Miller, C. P., and Bohnhoff, M., Development of streptomycin-resistant and streptomycin-dependent bacteria, in: Waksman, S. A., Streptomycin. Williams \& Wilkins Co., Baltimore, 1949, p. 158.

2. Hamburger, M., Berman, J. R., and Fabrizio, A., The development of streptomycin-resistant bacteria in the stools of patients treated for tuberculosis. J. Lab. \& Clin. Med., 1948, 33, 1460.

3. Parr, L. W., Sanitary significance of succession of coli-aerogenes organisms in fresh and in stored feces. Am. J. Pub. Health, 1936, 26, 39.

4. Weinstein, L., The spontaneous occurrence of new bacterial infections during the course of treatment with streptomycin or penicillin. Am. J. M. Sc., 1947, 214, 56.

5. Miller, C. P., and Bohnhoff, M., Effect of strepto- mycin therapy on the bacterial flora of the throat. Am. J. Med., 1949, 6, 417.

6. Yegian, D., and Vanderlinde, R. J., A quantitative analysis of the resistance of mycobacteria to streptomycin. J. Bact., 1948, 56, 177.

7. D'Esopo, N. D., Clinical significance of bacillary resistance in the treatment of pulmonary tuberculosis by streptomycin, in: Waksman, S. A., Streptomycin. Williams \& Wilkins Co., Baltimore, 1949, p. 349.

8. Pyle, M. M., Relative numbers of resistant tubercle bacilli in sputa of patients before and during treatment with streptomycin. Proc. of the Staff Meet., Mayo Clin., 1947, 22, 465.

9. Steenken, W., Jr., and Wolinsky, E., Resistance of tubercle bacilli to streptomycin, in: Waksman, S. A., Streptomycin. Williams \& Wilkins Co., Baltimore, 1949, p. 191. 